\title{
Relationship Between Patient-Reported Hospital Experience and 30-Day Mortality and Readmission Rates for Acute Myocardial Infarction, Heart Failure, and Pneumonia
}

\author{
Ning Dong, $M D, M S^{7}$, Jonathan D. Eisenberg, $M D^{2}$, Kumar Dharmarajan, $M D, M B A^{3,4,5}$, \\ Erica S. Spatz, MD, $\mathrm{MHS}^{3,4}$, and Nihar R. Desai, $M D, \mathrm{MPH}^{3,4}$
}

'Department of Medicine, Rutgers NJMS, Newark, NJ, USA; ${ }^{2}$ Department of Medicine, Yale School of Medicine, New Haven, CT, USA; ${ }^{3}$ Center for Outcomes Research and Evaluation, New Haven, CT, USA; ${ }^{4}$ Section of Cardiovascular Medicine, Yale School of Medicine, New Haven, CT, USA; ${ }^{5}$ Clover Health, Jersey City, NJ, USA.

KEY WORDS: patient experience; mortality; readmission.

J Gen Intern Med 34(4):526-8

DOI: $10.1007 / \mathrm{s} 11606-018-4746-6$

(c) Society of General Internal Medicine 2019

\section{INTRODUCTION}

The Hospital Consumer Assessment of Healthcare Providers and Systems (HCAHPS) survey has focused hospitals' attention on patient experience metrics. Hospitals' HCAHPS scores have been publicly reported since 2008 and more recently used as part of the Hospital Value-Based Purchasing (HVBP) program $^{1,2}$ as well as the CMS Overall Hospital Star Rating. ${ }^{3}$ As a component of the CMS Star Rating, HCAHPS scores carry significant weight in line with more traditional outcomes such as mortality and readmissions. ${ }^{3}$

However, little is known about the association of patient experience with mortality and readmissions. A direct association would support the use of patient experience metrics in evaluating hospitals, while an inverse association might suggest a tradeoff between patient experience and outcomes that would require specific consideration when comparing hospitals.

We sought to assess the relationship between hospital HCAHPS scores with hospital 30-day mortality and readmission rates for acute myocardial infarction (AMI), heart failure (HF), and pneumonia (conditions targeted by the HVBP program).

\section{METHODS}

Patient outcome and experience metrics were obtained from the Medicare Hospital Compare database at a hospital level. ${ }^{4}$ Outcome metrics included hospital 30-day risk-standardized mortality and readmission rates for AMI, HF, and PNA for the period between 7/1/2013 and 6/30/2016. Patient experience

Ning Dong and Jonathan D. Eisenberg are co-first authors

Published online February 11, 2019 metrics, derived from HCAHPS survey data collected between $10 / 1 / 2015$ and 9/30/2016, were divided into measures of overall satisfaction and nine individual domains of patient experience. Of note, hospital outcome measures reported on Hospital Compare are risk-standardized to account for differences in case mix by age and comorbidities.

Relationships between outcome and patient experience measures were assessed with pair-wise Pearson correlation coefficients (weighted by patient volume). Statistical analyses were performed using SAS (version 9.4).

\section{RESULTS}

\section{Study Sample}

The numbers of hospitals for mortality and readmission analyses were, respectively, 2346 and 2137 for AMI; 3212 and 3234 for HF; and 3316 and 3322 for PNA.

\section{Correlation Between Patient Experience and Outcome Measures}

The HCAHPS overall satisfaction measures were inversely correlated with readmission rates for all three conditions assessed $(r=-0.22$ to $-0.31, p<0.001)$ (Table 1$)$. The overall satisfaction measures were also inversely associated with mortality rates for AMI and PNA $(r=-0.10$ to $-0.20, p<0.001)$. However, no significant association was found with HF mortality.

All nine individual domains of patient experience (except quietness for AMI) were inversely correlated with readmission rates for AMI, HF, or pneumonia (Table 2). The strength of correlation was similar for all domains $(r=-0.16$ to -0.30 , $p<0.001)$ except quietness which had a weaker correlation.

In contrast, the correlations between the individual domains of patient experience and mortality were inconsistent and varied for different conditions. With AMI mortality, most domains were negatively correlated. With HF mortality, all nine domains of patient experience were positively correlated (except care transition), although the correlation was small 


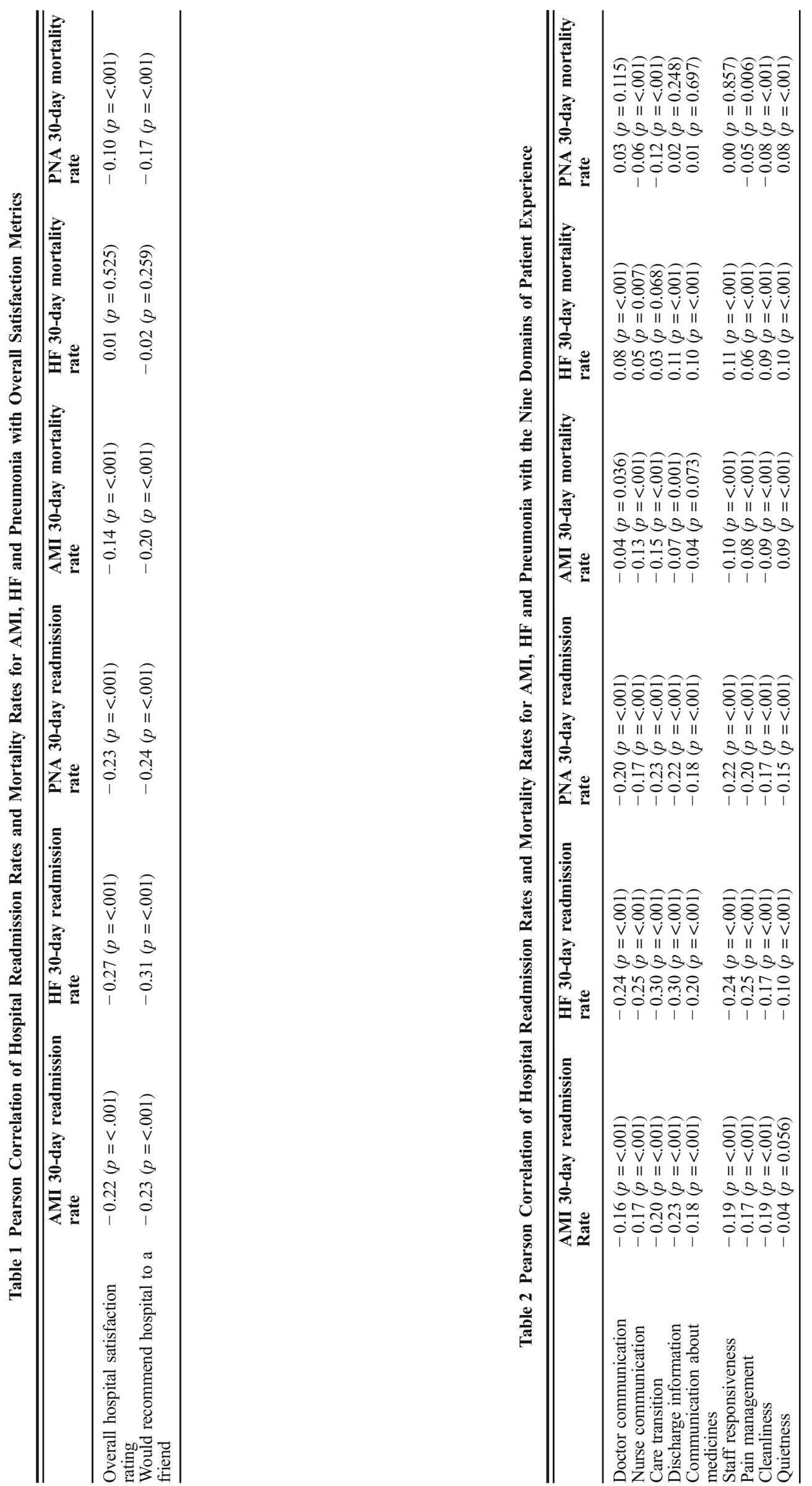


( $r=0.05$ to 0.11$)$. For PNA mortality, the correlations were mixed and either small or insignificant.

\section{DISCUSSION}

Using the CMS Hospital Compare dataset, we found that higher patient-reported overall satisfaction was associated with lower readmission rates for AMI, HF, and pneumonia, as well as lower mortality for AMI and pneumonia. Individual domains of patient experience were also inversely associated with readmissions for AMI, HF, and pneumonia, but mixed and inconsistent associations were found with mortality. Taken together, these results indicate an association between patient experience and outcomes which supports the use of patient experience metrics for assessing hospital quality.

We found a stronger and more consistent association between patient experience and hospital readmissions as compared with mortality. Readmissions are a complex and multifaceted challenge for hospital systems. It is intuitive that patient experience variables - which include communication regarding medicines and discharge plans - are integral to a successful discharge. In contrast, mortality may be driven more by illness severity. The lack of association between overall satisfaction and HF mortality is intriguing. The underlying cause is unclear, but may represent a difference in patient experience between acute and chronic conditions.

Our analysis suggests that patient-reported data can offer hospitals key insights into their performance, and may offer effective avenues for improving hospital outcomes. Additional work investigating the potential relationship between patient experience and outcomes needs to be undertaken to identify the direct mediators of this relationship.
Corresponding Author: Nihar R. Desai, MD, MPH; Section of Cardiovascular Medicine, Yale School of Medicine, 1 Church St., Suite 200, New Haven, CT 06510, USA (e-mail: nihar.desai@yale.edu).

\section{Compliance with Ethical Standards:}

Conflict of Interest: Dr. Kumar Dharmarajan is the Chief Scientific Officer of Clover Health, a Medicare Advantage company. Dr. Erica Spatz reports receiving support from the Centers for Medicare \& Medicaid Services to develop and maintain performance measures used in public reporting programs. Dr. Nihar Desai receives support from the Centers for Medicare \& Medicaid Services to develop and maintain performance measures, the American College of Cardiology Foundation, the Agency for Healthcare Research and Quality (K12 HSO2300O), and Johnson \& Johnson, through Yale University, to develop methods of clinical trial data sharing. All remaining authors declare that they do not have a conflict of interest.

Publisher's Note: Springer Nature remains neutral with regard to jurisdictional claims in published maps and institutional affiliations.

\section{REFERENCES}

1. HCAHPS Fact Sheet. Nov. 2017. Centers for Medicare \& Medicaid Services (CMS). Baltimore, MD USA. https://www.hcahpsonline.org/globalassets / hcahps/facts/hcahps_fact_sheet_november_2017.pdf Accessed 10/2018.

2. Tefera, L., Lehrman, W.G. and Conway P. Measurement of the Patient Experience: Clarifying Facts, Myths, and Approaches. N Engl J Med. 2016; 21244:E1-E2

3. Yale New Haven Health Services Corporation/Center for Outcomes Research \& Evaluation (YNHHSC/CORE). Overall Hospital Quality Star Ratings on Hospital Compare Methodology Report (v2.0). (May 2016) https://www.qualitynet.org/dcs/ContentServer? $\mathrm{c}=\mathrm{Page} \&$ pagename $=\mathrm{QnetPublic} \% 2 \mathrm{FPage} \% 2 \mathrm{FQnetTier} 2 \& \mathrm{ci}-$ $\mathrm{d}=1228775183434$. Retrieved 10/2018

4. Hospital Compare Data Archive. Centers for Medicare \& Medicaid Services (CMS). Baltimore, MD USA. https://data.medicare.gov/data/hospitalcompare. Published 7/2017. Accessed 10/2018. 